\title{
Preferential Looking in the Mentally Handicapped
}

\author{
${ }^{*}$ A. CHANDNA, †C. KARKI, †J. DAVIS, ${ }^{*}$ R. M. L. DORAN \\ Bristol
}

\begin{abstract}
Summary
We have assessed the feasibility of Preferential Looking (PL), using Teller Acuity Cards, for the estimation of binocular and monocular visual acuities in a group of mentally handicapped adults. Our results show the comparison between grating and recognition acuities, inter-observer variation, success rate, time taken and the sensitivity of this method in identifying monocular visual deficit in this group of subjects. The reasons for success or failure with PL methods in relation to criteria for mental handicap are discussed.
\end{abstract}

We are all familiar with the problems associated with the assessment of visual acuity and ocular status in the mentally handicapped. Similar difficulties of variable behaviour and inability to give verbal responses are encountered when dealing with preverbal children (children below the age of three years). Over the past decade Preferential Looking (PL) has been investigated in vision research laboratories as a means to determine the visual acuity of preverbal children,,$^{1-4}$ and is at present undergoing clinical evaluation..$^{5-7}$

Preferential Looking, a behavioural technique, is based on the principle that a subject (usually a young child) when presented with a patterned target, such as a high contrast grati$\mathrm{ng}$ in a uniform featureless environment, will prefer to look at the target as long as it is within its visual resolution capabilities. The method involves the sequential presentation of gratings in an increasing order of spatial frequency until the subject appears not to see the target and acuity is determined as the finest spatial frequency which repeatedly elicits a positive response.

This study was designed to assess the applicability of Preferential Looking in a group of adults with varying degrees of mental handicap and behavioural disorders. We investigated the comparison between grating (PL) and recognition acuity (Snellen), the success rate in obtaining reliable estimates of binocular and monocular acuity in one visit and the time taken for each test. Interobserver variation and the ability to identify monocular visual deficit was also assessed.

\section{Materials and Methods}

Teller Acuity Cards $\ddagger$ developed by Dr. D. Y. Teller at the University of Washington, U.S.A. were used. These cards have high contrast black and white gratings printed in a square patch on a plain grey background of

\footnotetext{
* University Department of Ophthalmology, Bristol Eye Hospital, Lower Maudlin Street, Bristol; and $\dagger$ Purdown Hospital, Purdown, Bristol.

This work was supported by a grant from the South Western Regional Health Authority and by the National Eye Research Centre, Bristol.

$\ddagger$ Teller acuity Cards obtainable from Vistech Consultants Inc; 1372 North Fairfield Road, Dayton, OHIO. 45432, USA.
}

Correspondence to: A. Chandna, D.O. F.R.C.S. Ed. Research Associate, Department of Ophthalmology, Bristol Eye Hospital, Lower Maudlin Street, Bristol BS1 2LX. 
equal average luminance. A standard set of 16 cards spans a frequency range from 0.32 to 38.0 cycles $/ \mathrm{cm}$ in half octave steps. ${ }^{*}$ The cards were displayed through an opening in a specially designed portable screen (the Bristol University Screen) (Fig. 1). Luminance was maintained above $1 \log \mathrm{cd} / \mathrm{m}^{2} .{ }^{8}$ As far as possible the test distance was maintained between $57 \mathrm{~cm}$ and $75 \mathrm{~cm}$. The distance of the subject from the screen was measured before and after the test. This was then averaged for each subject, $66 \mathrm{~cm}$ being the most common distance.

A modified staircase method of presentation was used to control the test. ${ }^{9}$ The cards were presented sequentially in an ascending or descending order of spatial frequency in a series of trials, as described in detail previously. $^{7}$

Two different staircases were used. In both, the test was initiated at the lowest spatial frequency i.e. $0.32 \mathrm{cy} / \mathrm{cm}$. Acuity threshold was determined as the highest spatial frequency at which the subject's correct responses were significantly above chance and at or below chance at the next higher spatial frequency. Between presentations the observer attracted the subject's attention to the centre of the opening of the screen.

Short Staircase (2 up; 1 down): The observer was masked to the grating position for the first presentation of each trial only. Having judged its location, the observer confirmed this by looking at the face of the card. If correct the card was rotated by 180 degrees and

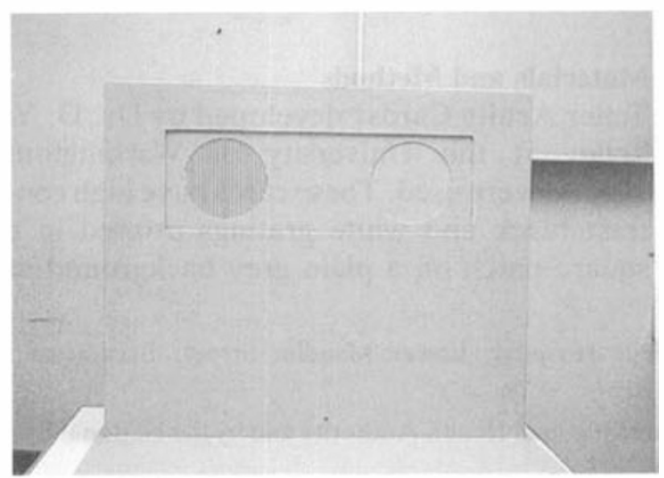

Fig. 1. Portable desktop screen for presenting Teller Acuity Cards. presented again in order to observe a shift of attention by the subject to the other side. If obtained, the grating one octave higher on the staircase was selected. If incorrect on either of these presentations the staircase descended.

Long Staircase (4 up; 2 down): The observer remained masked to the grating location throughout the test and a scorer conducted the test and recorded the results. Each trial consisted of five presentations. Four out of five correct responses led to a step up the scale by one octave. Two incorrect responses resulted in a step down the scale.

The response was either, observed through the central peephole of the card or the subject was encouraged to point at the gratings (Fig. 2). Some mentally handicapped subjects showed signs of anxiety when the observer disappeared behind the screen. With these individuals the test was conducted with the observer remaining in full view but masked to the location of the grating whenever required by the staircase.

\section{Subjects}

All subjects were residents at Purdown Hospital in Bristol. Due to historical reasons there is a male preponderance in the hospital and this is reflected in our subject groups.

Case selection was random, without prior knowledge of the degree of mental handicap, associated systemic abnormalities, ocular history, present ocular status or visual acuity. Monocular and binocular PL tests were

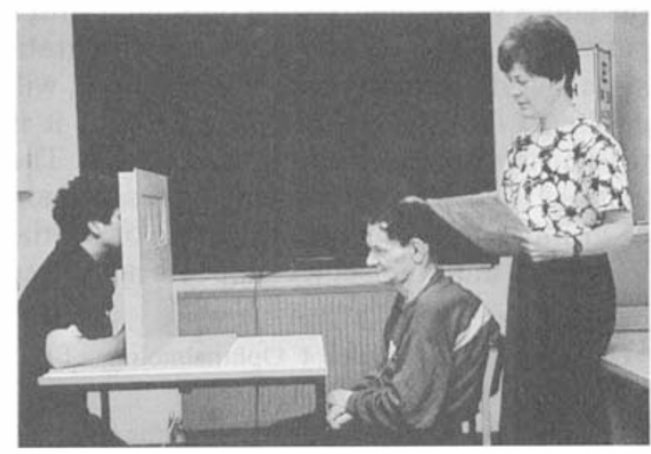

Fig. 2a. Subjects response observed through the central peephole in the acuity cards by the observer. An independent scorer provides a feedback.

* An octave is a doubling or halving of spatial frequency. 


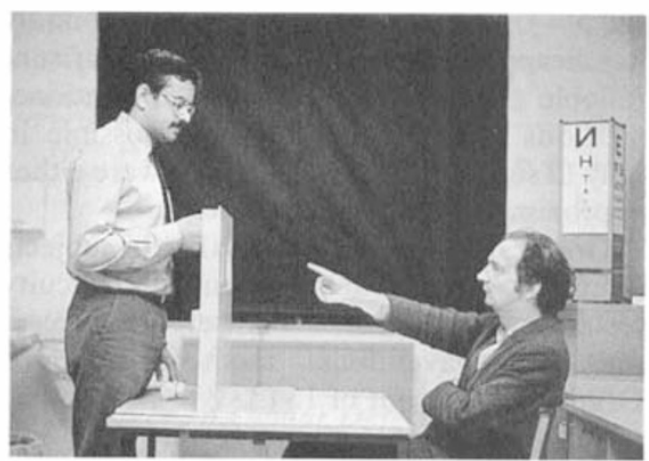

Fig. 2b. Subject being encouraged to point at the grating.

performed by an ophthalmologist (AC) with previous experience of PL methods but little experience of mentally handicapped patients.

For interobserver variation PL tests were carried out by a psychiatrist (CK) and a staff nurse (JD) with considerable experience of mentally handicapped patients but no previous experience of PL methods.

Group I: 15 subjects. (14 males, one female, age range $29-83$ years, mean age of 53.9 years) were selected from a group of residents in whom Snellen visual acuities had been recorded previously. Five subjects underwent binocular testing, five had binocular and monocular tests and five had only monocular PL acuity tests using the short staircase. Snellen visual acuities were recorded after the PL test.

Group II: 40 subjects (33 males, seven females; age range 24-81 years, mean age 49.7 years) were selected to achieve equal numbers, (ten in each subgroup), with varying degrees of mental handicap. Binocular acuity estimates were performed by Observer 1 (AC) using the long staircase. Twenty-three subjects were successfully tested in one visit. Seventeen subjects failed to give a reliable result on the first visit.

These 17 subjects were retested on a different occasion by Observer 1 using the short staircase. A third PL acuity test was performed by' a different observer-a 'key worker'. A key worker is a person who is actively involved in the daily care of the subject.

Twenty-two out of the 23 successfully tested subjects were recalled. One subject had been discharged. PL acuity tests with the long staircase were repeated either by Observer 2 (CK) or Observer 3 (JD) after being trained in the technique by Observer 1 (AC). The observers were masked from each other's results.

Group III: A further 15 subjects (nine males, six females; age range 29-82 years, mean age 54 years) were selected randomly, with mental handicaps similar in range to the 23 successful subjects in the previous group. Monocular PL acuity tests were carried out to assess the feasibility of monocular tests and the sensitivity of PL in identifying ocular defect.

Each subject had an ocular examination after the PL test which included ocular history as gathered from case notes, cover test, ocular movements, pupillary reflexes, slit lamp examination of the anterior segment wherever possible, or failing which a penlight examination. The optic disc and macular area were examined through an undilated pupil.

Visual acuity was estimated by conventional methods (e.g. Catford Drum) by an orthoptist unfamiliar with the PL acuity results.

\section{Results}

\section{Comparison Between Grating (PL) and}

Recognition Acuity-(Snellen Acuity; Group

\section{Subjects)}

Figure 3 shows the correlation between the monocular and binocular acuity estimates, obtained by PL methods plotted against Snellen acuities. The line corresponds to the points at which recognition and grating acuities are in perfect agreement. (Corr. RE: 0.93. LE: 0.74. Binocular 0.48). Ninety-five per cent of the monocular and $100 \%$ of binocular acuity measures of grating acuity were within 0.5 octaves of recognition acuity values. There was no evidence for over $(5=+0.5)$ or under $(4=-0.5 \& 1=-1.0)$ estimation of acuity by PL for monocular results but some evidence of a trend towards under-estimation of acuity by PL for binocular results $(2=0 ; 8=-0.5$ octaves $)$.

Another method of examining the extent of agreement between the values obtained by the two methods, ${ }^{10}$ is seen in Figure 4, where for each acuity measured the difference 


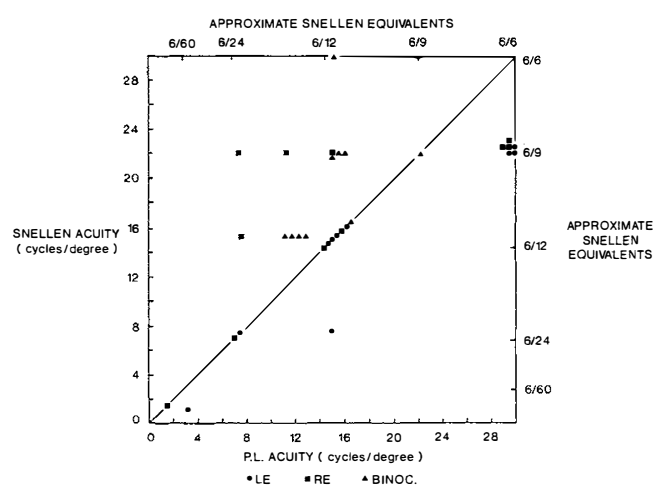

Fig. 3. Comparison between grating $(P L)$ and recognition (Snellen) acuity.

between grating and recognition acuity is plotted against the mean acuity. 4.5 cycles per degree corresponds to the mean difference between two adjacent lines on a standard Snellen chart and $16(51.6 \%)$ differences fall within this range. However, if twice the mean difference between the two adjacent Snellen lines were considered the extent of agreement increases to $29(93.5 \%)$.

\section{Success Rate}

We have defined success as obtaining reliable acuity estimates by our PL method in one visit.

Group II-For the 40 subjects with varying degrees of mental handicap, $23(57.5 \%)$ were tested successfully for binocular acuity estimates. The successful subjects had mild $(\mathrm{N}=4)$, moderate $(\mathrm{N}=11)$, or severe $(\mathrm{N}=7)$ degrees of mental handicap. Only one subject had profound mental handicap. In contrast, the 17 unsuccessful patients

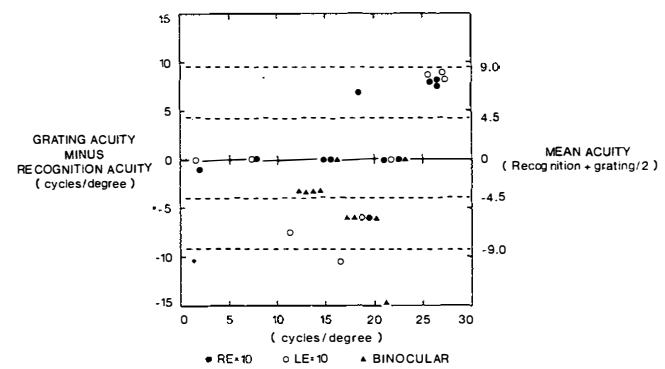

Fig. 4. Extent of agreement between PL and Snellen acuities.
$(42.5 \%)$, were severely $(\mathrm{N}=3)$ or profoundly handicapped $(\mathrm{N}=14)$. In comparison, reliable estimates of vision by conventional methods (Catford Drum) were possible in only 13 subjects $(32.5 \%)$. The rest were either approximations or not possible.

Group III-Ten out of a further 15 subjects $(66.6 \%)$, gave reliable monocular acuity results with the short staircase. The success rate for conventional methods (Catford Drum) was five out of $15(33.3 \%)$.

All subjects in Groups II and III were unable to cooperate with the recognition acuity method (Snellen Chart).

\section{Interobserver Variation}

Figure 5 shows the extent of correlation of acuity estimates obtained on 21 of the 22 subjects. One subject failed to cooperate. The results for Observer 2 (14 tests) and Observer 3 (7 tests) are plotted against those obtained by Observer 1 (Corr. $=0.61$ ).

The extent of acuity difference between Observer 1 (PL acuity test 1) and Observers 2 and 3 (PL acuity test 2 ) is seen in Figure 6. Nineteen $(90.5 \%)$ points fall within the 0.5 octave and all $21(100 \%)$ within the 1.0 octave limit.

\section{Test Time}

Binocular test times (long staircase) for Observer 1 ranged from five to ten minutes $(\mathrm{N}=23)$, and averaged 6.9 minutes. For Observers 2 and 3 test time ranged from five to ten minutes $(\mathrm{N}=21)$, with the same average. Monocular test times (short staircase) ranged from three to six minutes $(\mathrm{N}=10)$, average 4.11 minutes per eye.

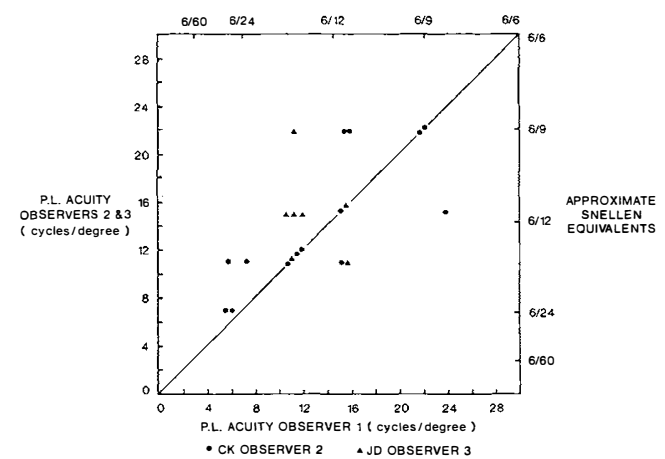

Fig. 5. Correlation between binocular acuity results obtained by Observers 1, 2 and 3. 


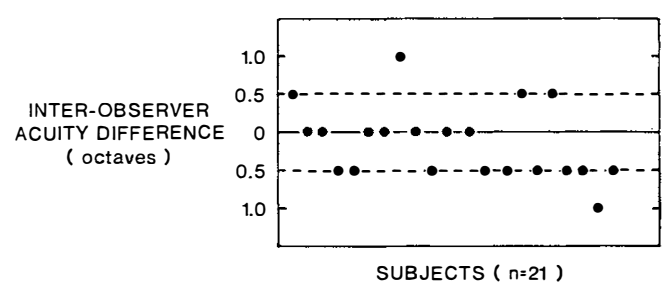

Fig. 6. Interobserver variation: Interobserver acuity difference plotted for each subject.

\section{Identification of Ocular Defect}

Subsequent ocular examination revealed significant ocular defects in nine subjects (5; Group I and Group III). All such defects were readily identified by monocular PL acuity tests (Fig. 7). An interocular acuity difference of 0.5 octave or more was considered significant.

\section{Discussion}

There are only a few reports in the literature regarding the incidence of ocular abnormalities in the mentally handicapped. ${ }^{11}$ As far as we are aware there are no reports on the assessment of visual acuity in mentally impaired adults. There are some reports regarding the use of PL methods in mentally handicapped and neurologically impaired children. ${ }^{6,12-15}$ We feel there is a need for a reliable and practical method for the estimation of visual acuity in mentally handicapped adults.

Our study was concerned with the practicality of PL methods in clinical practice when applied to the mentally handicapped. The initial validation required the comparison with the standard recognition task of Snellen acuity in those able to perform both tests. A good correlation was revealed and the extent of agreement compares well with that obtained by Mosley et al, ${ }^{16}$ at the two standard deviation ( 9.0 cycles/degree) level. It does not compare well at the 4.5 cycles/degree limit. This may be due to the extremely variable attention span even in the subjects with moderate mental handicap. The interobserver variation results failed to reveal any significant difference between the acuity estimates obtained by an ophthalmologist with experience of PL methods and observers without previous experiences of PL (An interobserver difference of 0.5 octave was considered not significant. ${ }^{5,7}$ ) The training session was completed in one afternoon. The lack of significant difference confirms the robustness of the method which may well be used by persons closely involved with the mentally handicapped.

The success or failure in obtaining a reliable acuity estimate was further analysed in relation to mental age, IQ rating and the Wessex Assessment Score (Table I). Wessex Assessment is based on an interview procedure to estimate the self-help skills and associated behavioural problems in the mentally handicapped. This classification was mainly designed to assess the feasibility of community placement for the mentally handicapped. Groups 1-4 are in increasing order of dependence and behavioural disturbance. Mental age and IQ ratings were not available for all the subjects. The reasons for this were mainly lack of cooperation.

The range of mental age and IQ ratings does not appear to be different for successful and unsuccessful subjects. In the unsuccessful group, an IQ rating of 48 was obtained by only one subject. However the mean values of the two groups showed significant differences. (Mean mental age of 5.5 years for the successful and 2.7 years for the unsuccessful patients). Mean IQ ratings were 34.28 for the

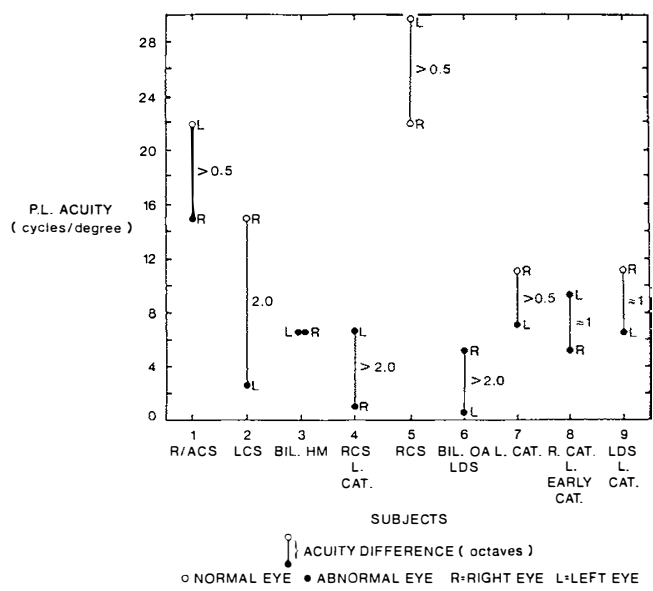

Fig. 7. Identification of ocular defect by monocular PL tests. 1. right/alternating convergent squint. 2. Left convergent squint. 3. Bilateral hypermetropia. 4. Right convergent squint. Left cataract. 5. Right convergent squint. 6. Bilateral optic atrophy. Left convergent squint. 7. Left cataract. 8. Right cataract. Left early lens opacities. 9. Left divergent squint. Left cataract. 
Table I

\begin{tabular}{|c|c|c|c|c|c|c|c|}
\hline \multirow[b]{2}{*}{ Patients } & \multicolumn{3}{|c|}{ P.L. and mental handicap } & \multicolumn{4}{|c|}{ Wessex assessment } \\
\hline & $\stackrel{\mathrm{N}}{\text { Total no. } 55}$ & $\begin{array}{c}\text { M.A. } \\
\text { Range-yrs }\end{array}$ & $\begin{array}{l}\text { I.Q. rating } \\
\text { Range }\end{array}$ & Group & $\mathbf{N}$ & $\%$ & \\
\hline Successful & 34 & $\begin{array}{c}2.0-7.6 \\
(\text { mean } 5.5)\end{array}$ & $\begin{array}{c}18-54 \\
(\text { mean } 34.28)\end{array}$ & $\begin{array}{l}1 \\
2 \\
3 \\
4\end{array}$ & $\begin{array}{r}15 \\
16 \\
2 \\
1\end{array}$ & $\begin{array}{r}45 \\
46 \\
6 \\
2\end{array}$ & $\begin{array}{r}91 \% \\
8 \%\end{array}$ \\
\hline Unsuccessful & 21 & $\begin{array}{c}1.0-8.0 \\
(\text { mean } 2.7)\end{array}$ & $\begin{array}{c}0-48^{*} \\
(\text { mean } 19.85)\end{array}$ & $\begin{array}{l}1 \\
2 \\
3 \\
4\end{array}$ & $\begin{array}{r}1 \\
5 \\
5 \\
10\end{array}$ & $\begin{array}{r}4 \\
23 \\
24 \\
48\end{array}$ & $\begin{array}{l}27 \% \\
72 \%\end{array}$ \\
\hline
\end{tabular}

MA $=$ Mental Age.

* IQ of 48 was obtained in only one subject.

successful and 19.85 for the unsuccessful group. These figures show distinct differences although the numbers are small. In relation to Wessex score (data available on all residents) we find a majority of successful subjects in Groups 1 and 2. The unsuccessful patients were mainly found to belong to Wessex Groups 3 and 4, which are associated with behavioural disorders. This is in agreement with the behavioural nature of PL as an acuity test. It is also significant that a reliable ocular examination was not possible in any of the 17 unsuccessful subjects in Group II. This overall lack of success in the assessment of ocular status and visual acuity tends to identify a group of subjects who are 'not testable'. This did not change even when retested by Observer 1 and a key worker.

The success rate was further confirmed by repeat PL testing for the successful group. Twenty-one out of the 22 subjects gave reliable results. The higher success rate for monocular tests is probably due to the different staircase procedure (short staircase). For all subjects the success rate was worse with the Catford Drum.

The time taken for each PL acuity test is comparable with the test times when this method is applied for acuity estimation of preverbal children. ${ }^{7}$ The short staircase offers a practical method considering the short concentration span in this population group.

The sensitivity of this technique is borne out by the identification of monocular visual deficits in all nine subjects who were found to have ocular abnormalities on subsequent examination.

We felt that the procedure should be modified to suit the behavioural needs of the mentally impaired. A desktop version and the observer remaining in full view when conducting the test reassures the subject and increases cooperation.

We wish to thank, Dr. C. Bartlett, M.R.C. Psych., Consultant Psychiatrist, Purdown Hospital, Bristol for her help and advice, Ms. C. Aitchison for measuring visual acuity with conventional methods, and the residents of Purdown Hospital who participated in the study.

\section{References}

${ }^{1}$ Fantz RL, Ordy JM, Udelf MS: Maturation of pattern vision in infants during the first six months. J Comp Physiol Psych 1962, 55: 907-17.

${ }^{2}$ Teller DY: The forced choice preferential looking technique for use with human infants. Infant Behaviour and Development 1979, 2: 135-53.

${ }^{3}$ Mayer DL and Dobson V: Visual acuity development in infants and young children as assessed by operant preferential looking. Vision Res 1982, 22: 1141-51.

${ }^{4}$ Atkinson J, Braddick O, Pimm-Smith E: 'Preferential looking' for monocular and binocular acuity testing for infants. Br J Ophthalmol 1982 , 66: $264-8$.

${ }^{5}$ Teller DY, McDonald MA, Preston K, et al. Assessment of visual acuity in infants and young children. The Acuity Card Procedure. Dev Med Child Neurol 1986, 28: 779-98.

${ }^{6}$ Mohn G and Van Hof-van Duin J: Rapid Assessment in infants and children in a clinical setting, 
using acuity cards. Doc Ophthalom Proc 1986, 45: 363-71.

${ }^{7}$ Chandna A, Pearson CM, Doran RML: Preferential Looking in Clinical Practice: A Years Experience. EYE 1988 (In Press).

${ }^{8}$ Dobson V: Clinical applications of Preferential looking measures of visual acuity. Behav Brain Res 1983, 19: 25-38.

${ }^{9}$ Cornsweet TN: The staircase method in psychophysics. Am. J. Pyschol 1962, 75: 485-91.

${ }^{10}$ Bland JM and Altman DG: Statistical method for assessing agreement between two methods of clinical measurement. Lancet 1986, i: 307-10.

${ }^{11}$ Donoghue EC, Abbas KA, Gal E: The medical assessment of mentally retarded children in hospital. Br J Psych 1970, 117: 531-2.
${ }^{12}$ Hertz BG: Acuity Card testing of retarded children. Behav. Brain Res. 1987, 24: 85-92.

${ }^{13}$ Duckman RH and Selenow A: Use of forced preferential looking for measurement of visual acuity in a population of neurologically impaired children. Am J Optom Physiol Opt 1983, 60: 817-21.

${ }^{14}$ Jenkins PL, Simon JW, Kandel GL, et al: A simple grating visual acuity test for impaired children. Am. J. Ophthalmol. 1985, 99: 652-8.

${ }^{15}$ Lennerstrand G, Axelsson A, Anderson G: Visual assessment of preferential looking techniques in mentally retarded children. Acta Ophthalmol 1983, 61: 183-5.

${ }^{16}$ Moseley MJ, Fielder AR, Thompson JR, et al: Grating and recognition acuities of young amblyopes. Brit J Ophthalmol 1988, 72: 50-4. 